\title{
Treatment outcomes of implant-assisted removable partial denture with distal extension based on the Kennedy classification and attachment type: a systematic review
}

Adityakrisna Yoshi Putra Wigianto, Takaharu Goto* ${ }^{*}$, Yuki Iwawaki, Yuichi Ishida, Megumi Watanabe and Tetsuo Ichikawa

\begin{abstract}
Background: Implant-assisted removable partial dentures (IARPDs) have recently become popular, but little information is available on the treatment outcomes based on the Kennedy classification and attachment types.

Objective: The objective of this review was to evaluate the treatment outcomes of IARPD delivered for distal extension edentulous areas based on the differences in the Kennedy classification and attachment type.

Materials and methods: English-language clinical studies on IARPD published between January 1980 and February 2020 were collected from MEDLINE (via PubMed), the Cochrane Library (via the Cochrane Central Register of Controlled Trials), Scopus online database, and manual searching. Two reviewers selected the articles based on pre-determined inclusion and exclusion criteria, followed by data extraction and analysis.

Results: Eighty-one studies were selected after evaluating the titles and abstracts of 2410 papers. Nineteen studies were finally included after the perusal of the full text. Fourteen studies focused on Class I, 4 studies investigated both Class I and II, and only 1 study was conducted on Kennedy's class II. Eight types of attachments were reported. The ball attachment was the most frequently used attachment, which was employed in 8 of the included studies. The implant survival rate ranged from 91 to 100\%. The reported marginal bone loss ranged from $0.3 \mathrm{~mm}$ to $2.30 \mathrm{~mm}$. The patient satisfaction was higher with IARPD than with conventional RPDs or that before treatment. The results of prosthetic complications were heterogeneous and inconclusive.

Conclusion: IARPD exhibited favorable clinical outcomes when used as a replacement for distal extension edentulous areas. The comparison between the clinical outcomes of Kennedy's class I and II was inconclusive owing to the lack of studies focusing on Kennedy Class II alone. The stud attachment was the most commonly used type in IARPDs. Overall, the different attachment systems did not influence the implant survival rate and patient satisfaction. Further high-quality studies are needed to investigate the attachment systems used in IARPD.
\end{abstract}

Keywords: Dental implant, Implant-assisted removable partial denture, Kennedy classification, Attachment, Treatment outcomes

*Correspondence: tak510@tokushima-u.ac.jp

Department of Prosthodontics \& Oral Rehabilitation, Tokushima

University Graduate School of Biomedical Sciences, 3-18-15 Kuramoto, Tokushima 770-8504, Japan

\begin{abstract}
Introduction
At the outset, osseointegrated implants were used to support bone anchored-bridges in patients with complete edentulism, and the application of implant-based prostheses was gradually extended to partial edentulism.
\end{abstract}


Currently, implant-based prostheses can be used for the rehabilitation of all types of edentulous spans with predictable outcomes.

Dental implants have also been used widely to support overdentures, leading to extensive evaluations for decades. The McGill consensus statement on overdentures published in 2002 stated that mandibular two-implant overdentures are the first choice of treatment for completely edentulous patients [1]. This treatment modality improves denture retention and stability with the help of a few osseointegrated implants, which also helps to limit the treatment cost [2]. Subsequently, the application of dental implants to support overdentures was expanded to removable partial dentures (RPD). Several current epidemiological studies have reported a decrease in the frequency of complete edentulism, which is expected to exhibit a declining trend in future years, owing to the increase in the availability of dental healthcare facilities. Thus, the number of patients requiring RPD treatment has increased $[3,4]$. The frequency for RPD treatment is the highest for Kennedy's class I, followed by Kennedy's class II [5]. However, the prosthodontic replacement of these types of edentulous arches is beset by several challenges such as unfavorable movements of the RPD due to the differences between the viscoelasticity of the oral mucosa and abutment teeth, retention loss, mucosal irritation or ulceration, and discomfort arising from the retentive clasps $[6,7]$. Implant-assisted RPDs (IARPDs) are a viable option that can overcome the abovementioned issues and limitations of conventional RPDs (CRPDs) [7]. The use of an implant to assist the RPD confers some advantages, such as improved retention, stability, patient comfort, patient satisfaction, confidence, reduction of denture movement under the fulcrum line, decreased requirement for relining, and reduced risk of combination syndrome $[8,9]$. Several studies have investigated the clinical outcomes and viability of IARPD. Several review studies have also been published on this topic. De Freitas RF et al. reported on the patient satisfaction, survival rate of implants, and prosthetic complications of mandibular IARPDs in 2012 [10]. Subsequently, numerous studies have reported on this aspect, including various case reports. A systematic review and meta-analysis conducted by Park et al. evaluated the treatment outcome after replacing the CRPD with IARPD in patients with Kennedy's class I in the mandibular arch [11]. However, from the clinical perspective, Kennedy's class II is as important as Kennedy's class I.

Moreover, various types of attachment systems are used in IARPDs. The selection of these attachments is usually based on several considerations, such as the amount of retention needed, inter-arch space, patient dexterity, and the clinician's skill [12]. Aldhohrah et al. conducted a systematic review and meta-analysis on different attachment systems used in mandibular implant overdentures (IOD) [13]. The available attachment types used in IARPD are similar to those used in IOD, but the biomechanical conditions of the two prostheses are not the same. However, no study has investigated the different types of attachment used against the prosthesis survival rates and other clinical parameters in patients with Kennedy's Class I and II treated with IARPD.

This systematic review was conducted to evaluate the treatment outcomes of IARPD with distal extension based on the differences in the Kennedy classification and attachment type, in addition to a comprehensive evaluation of the latest findings on this topic.

\section{Materials and methods}

This systematic review was conducted according to the Preferred Reporting Items for Systematic Reviews and Meta-analysis (PRISMA) [14] with following the PICO $(P=$ patient problem/population, $I=$ intervention, $C=$ comparison, $O=$ outcomes) model:

- Population: patients with Kennedy classification I or II either on maxilla or mandibula

- Intervention: Implant Assisted Removable Partial Dentures

- Comparison: Kennedy classification and attachment system

- Outcome: clinical outcomes, such as implant survival rate, marginal bone loss, patient satisfaction.

\section{Information sources and search}

The English language literature published between January 1980 and February 2020 were extracted using the MEDLINE (via PubMed), Cochrane Library (via Cochrane Central Register of Controlled Trials, CENTRAL), and Scopus databases. The electronic database search was performed using keywords and MeSH terms based on the following search strategy used for exploring MEDLINE (via PubMed): (("Denture, Partial"[Mesh]) AND ("Dental Prosthesis, Implant-Supported"[Mesh] OR "Dental Implants"[Mesh])) OR (implant-assisted removable partial denture [Title/Abstract]). A manual search was also performed in addition to these database searches by checking the bibliography of all identified articles for potentially relevant additional studies.

\section{Inclusion criteria}

The studies were selected based on the following inclusion criteria: (1) studies with patients with Kennedy's class I or II (treated with distal extension RPD); (2) case reports, cohort studies, or randomized controlled trials 
(RCTs); (3) studies that reported clinical outcomes, such as marginal bone loss or survival rate of implants, periodontal conditions of the abutment teeth, or patient satisfaction; (4) studies in which the attachment system was clearly described; and (5) studies whose full-text was available in English.

\section{Exclusion criteria}

The following studies were excluded: in vitro studies, animal studies, and review studies, studies that did not mention the method of measuring clinical outcomes, and those reporting qualitative outcomes descriptions without presenting the exact values.

\section{Study selection}

Figure 1 demonstrates the literature search strategy used in this study. Two authors (T.G. and A.Y.P.W.) who had previously determined the criteria independently evaluated the literature search. First, the collected titles and abstracts were selected according to the aim and pre-determined criteria. Second, two reviewers confirmed the concurrence of the results, and the full-text of these articles was read to further examine the details of the results reported. Subsequently, the discrepancies in the results of the two authors were discussed with a third reviewer (T.I.). Finally, the studies that investigated the prognosis and IARPD outcomes with respect to the attachment systems were included.

\section{Data collection process and data items}

An extraction sheet was created using Microsoft Excel (Microsoft Office Professional Plus 2019, CA, USA) for data collection. The table contained the following information: author, publication year, research design, follow-up period, number of patients, number of implants, attachment design, and results. The literature review was performed after summarizing the results based on each subfield.

\section{Results \\ Study selection}

A total of 2410 studies published between 1986 and 2020 were obtained according to the search strategy described in Fig. 1. Eighty-one studies were selected for fulltext assessment after initial screening of the titles and abstracts. Nineteen studies were finally selected after the application of the inclusion criteria. Based on the year of publication, the earliest included study was published in 2003[15]. Three studies were from the 2000s [15-17], and 16 studies were from the 2010s and 2020 [18-33]. Three RCTs had the highest level of evidence with respect to the study design [25-27], followed by 2 randomized crossover trials [16, 31], 9 prospective studies [18-24, 30, $33], 2$ retrospective studies [15, 28], and 3 case reports $[17,25,29]$.

The current review focused on two Kennedy classifications, class I and II. Most of the studies were based

\section{Literature search with keywords}

"Denture, Partial"[Mesh], "Dental Prosthesis, Implant-Supported"[Mesh]

Dental Implants"[Mesh], Implant-assisted removable partial denture[Title/Abstract]

Number of selected papers: 2410

Assessments by titles and abstracts

Number of selected papers: 81

Assessments by full-text articles

19 papers and 15 clinical parameters included in this literature review

-Implant survival rate: 10 papers $\cdot$ Masticatory performance: 1 paper

- Mean bone loss:

7 papers -Mucosal health:

1 paper

- Occlusal contact area:

2 papers - Plaque score:

1 paper

- Occlusal force:

2 papers - Maximum bite force:

1 paper

- Patient satisfaction:

8 papers - Food comminution index: 1 paper

- Prosthetic complication:

- Bleeding on probing:

4 papers $\cdot$ Swallowing threshold:

3 papers - Energy intake:

1 paper

- Implant mobility:

1 paper

Fig. 1 Literature review strategy 
only on class I [16, 19, 20, 22-28, 30-33], followed by studies that included both class I and II cases (4 studies) $[15,18,21,29]$, and only one study investigated class II cases[17]. The treatment outcomes were not described separately or compared between these 2 classes in the four studies that included both classes.

Eight different types of denture attachments were utilized in the included studies. The ball attachment was the most frequently used attachment (8) $[19,20,22-$ $24,28,30,33]$, followed by the locator (5) [17, 18, 26, $27,29]$, healing abutment $[15,16]$, equator [32], stress breaking ball [31], ball with clix [21], extra-coronal resilient attachment (ERA) [25], and resilient attachment [15]. However, the treatment outcomes between those different attachments were compared only in one study [15]. Fifteen clinical parameters were extracted from the included studies.

This review also attempted to include published IARPD studies that dealt with the maxillary and mandibular arches. Nevertheless, we did not find a single study that evaluated the use of IARPD in the maxillary arch only. A majority of the included studies dealt only with the mandibular arch [15-17, 19-22, 24-28, 30-33]; meanwhile, only 3 studies included both maxillary and mandibular arches [18, 23, 29].

\section{Clinical outcomes}

The characteristics of the selected studies are summarized in Table 1 . The treatment outcomes of studies that investigated only Kennedy's class I or class II were implant survival rate and marginal bone loss. The comparison of these two clinical outcomes based on Kennedy's classification is presented in Figs. 2 and 3.

\section{Implant survival rates}

Overall, the implant survival rates reported by the included studies ranged from $91 \%$ to $100 \%$. Jensen et al. reported a $100 \%$ implant survival rate after 3 months of evaluation [26]. Similar survival rates $(100 \%)$ were also reported by Grageda et al. [25], Turkyilmaz [17], and Mitrani et al. [15] after a mean evaluation period of 3 years, 18 months, and 2.52 years, respectively. Payne et al. incorporated the longest evaluation period (10 years), which yielded a 92\% survival rate [30]. The lowest survival rate (91.6\%) was reported by Ortiz-Puigpelat et al., who evaluated in 12 patients for a mean duration of 28.6 months [29]. All studies reported high implant survival rates, irrespective of the attachment type. The implant survival rates of the included studies (Fig. 2) based on the Kennedy classification were as follows: class I, 91.7-100\%; class II, 100\%; and classes I and II, 91.6-100\%.

\section{Marginal bone loss}

Eight studies included in this review reported the mean bone loss data, with the reported mean value ranging from $0.3 \mathrm{~mm}$ to $2.30 \mathrm{~mm}$. Only one study by Threeburuth et al. compared the use of mini-implants with conventional implants and reported significantly lower marginal bone loss with the mini-implant with equator attachment group [33]. Mitrani et al. compared the healing outcomes between the healing abutment and resilient attachments [15]. However, other studies did not report any significant differences in the outcomes with respect to the different implant types, positions, or attachment systems. The highest marginal bone loss was reported by Payne et al., which was as high as $2.20 \pm 0.81 \mathrm{~mm}$ [30]. The different types of attachments did not specifically increase or decrease the marginal bone loss. Figure 3 depicts the comparison between the different Kennedy classes (mean values, class I: $0.47-2.20 \mathrm{~mm}$, class II: $0.3 \mathrm{~mm}$, and classes I and II: $0.32-1.04 \mathrm{~mm}$ ).

\section{Patient satisfaction}

Eight of the 19 studies included in this review reported patient satisfaction outcomes using precise values and pre-determined parameters. Six studies, which were conducted by Jensen et al. [27], Campos et al. [19], OrtizPuigpelat et al. [29], Goncalves et al. [23], Wismeijer et al. [33], and Ohkubo et al. [16] compared the differences between the implant-supported RPDs (ISRPD)/ IARPD and CRPD. All studies stated that patients reported significantly better satisfaction with ISRPD/ IARPD over CRPD. Threeburuth et al. [32] and Mitrani et al. [15] compared this outcome between before and after ISRPD/IARPD treatment, and both studies found a significant increase in patient satisfaction after treatment. However, Threeburuth et al. found no significant difference between the use of mini-implants and conventional implants [32]. IARPD treatment increased patient satisfaction compared to that before treatment or CRPD usage, irrespective of the attachment types and Kennedy's classification.

\section{Technical/prosthetic complications}

Three of the included studies reported the prosthetic/ technical complication outcomes for IARPDs [21, $28,29]$. Jensen et al. reported that 15 of 23 prostheses used ball attachments did not have complications [28]. Ortiz-Puigpelat et al. did not find any locator abutment loosening; however, all plastic retentive components (matrix) had to be changed after 12 months. Some other complications were also observed [29]. Gates et al. used a ball with a clip attachment and reported that one prosthesis needed attachment replacement, 


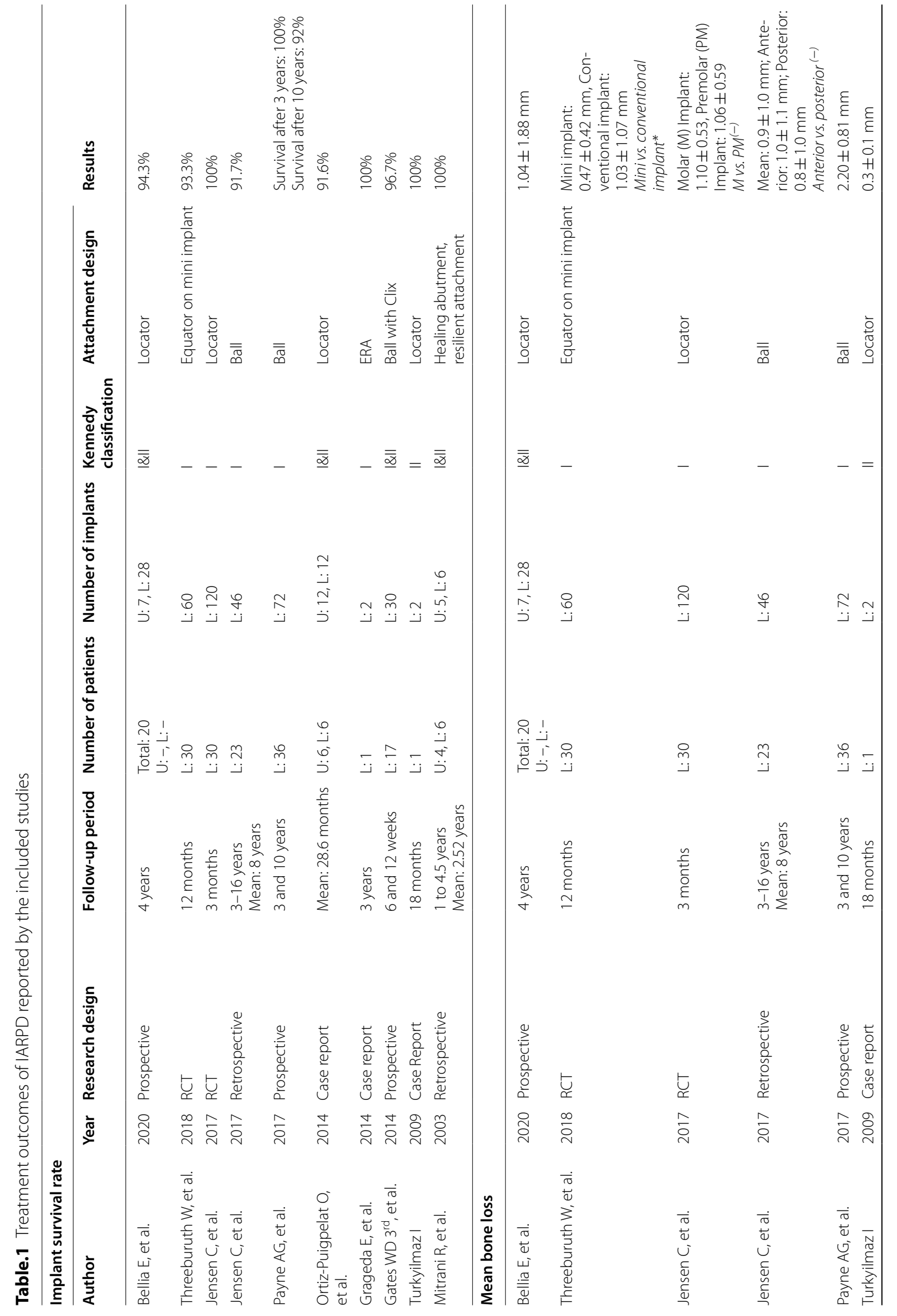




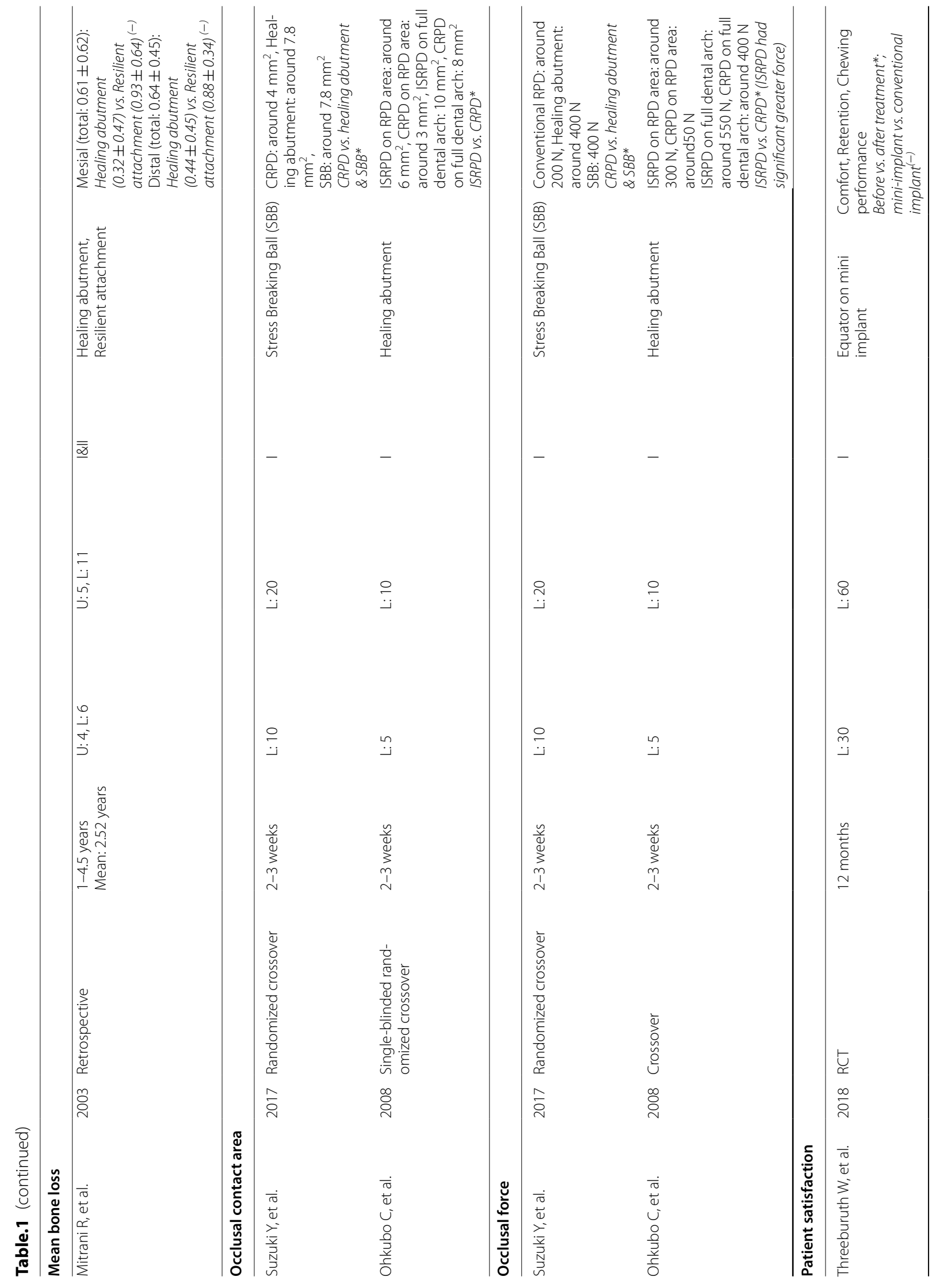




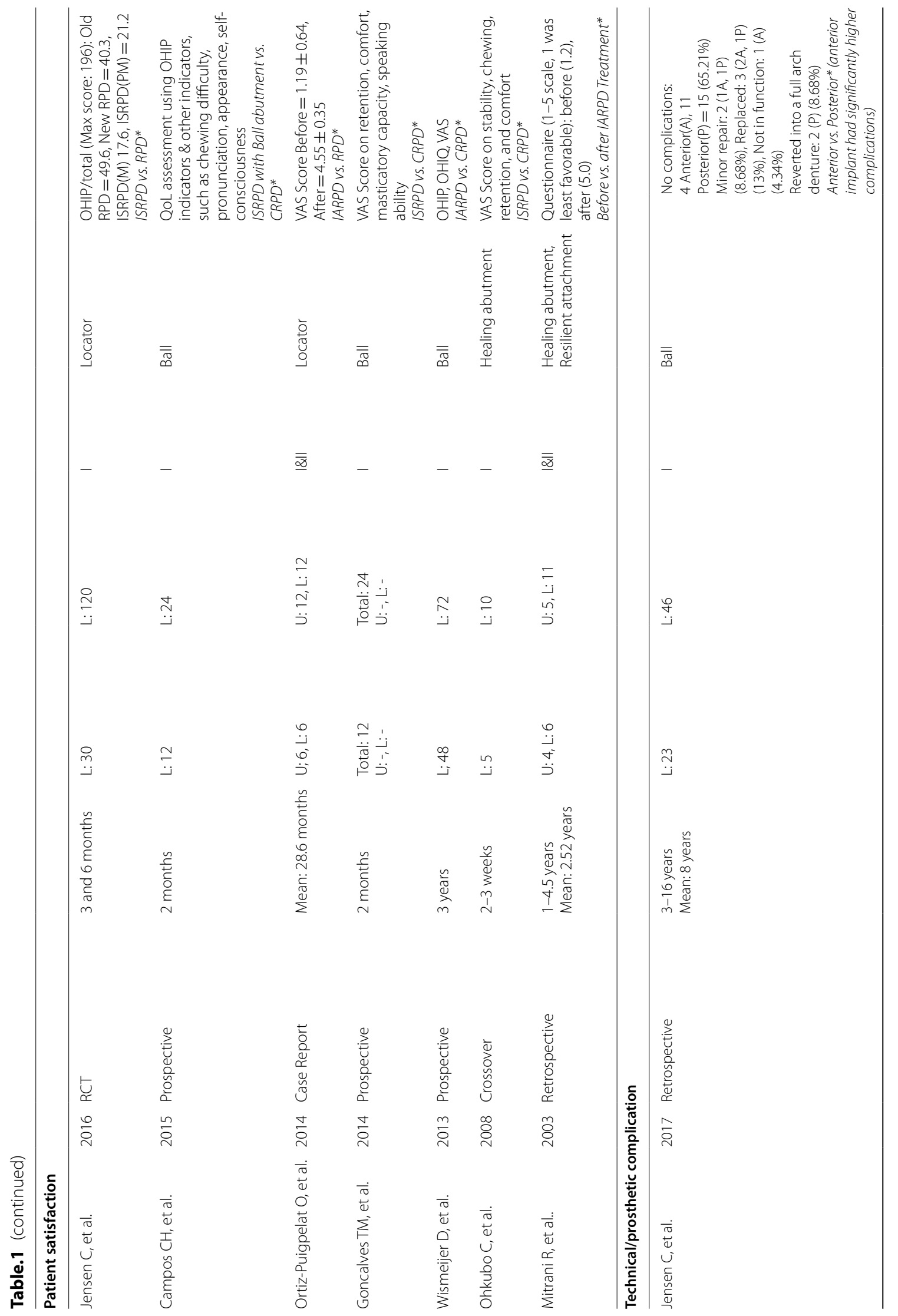




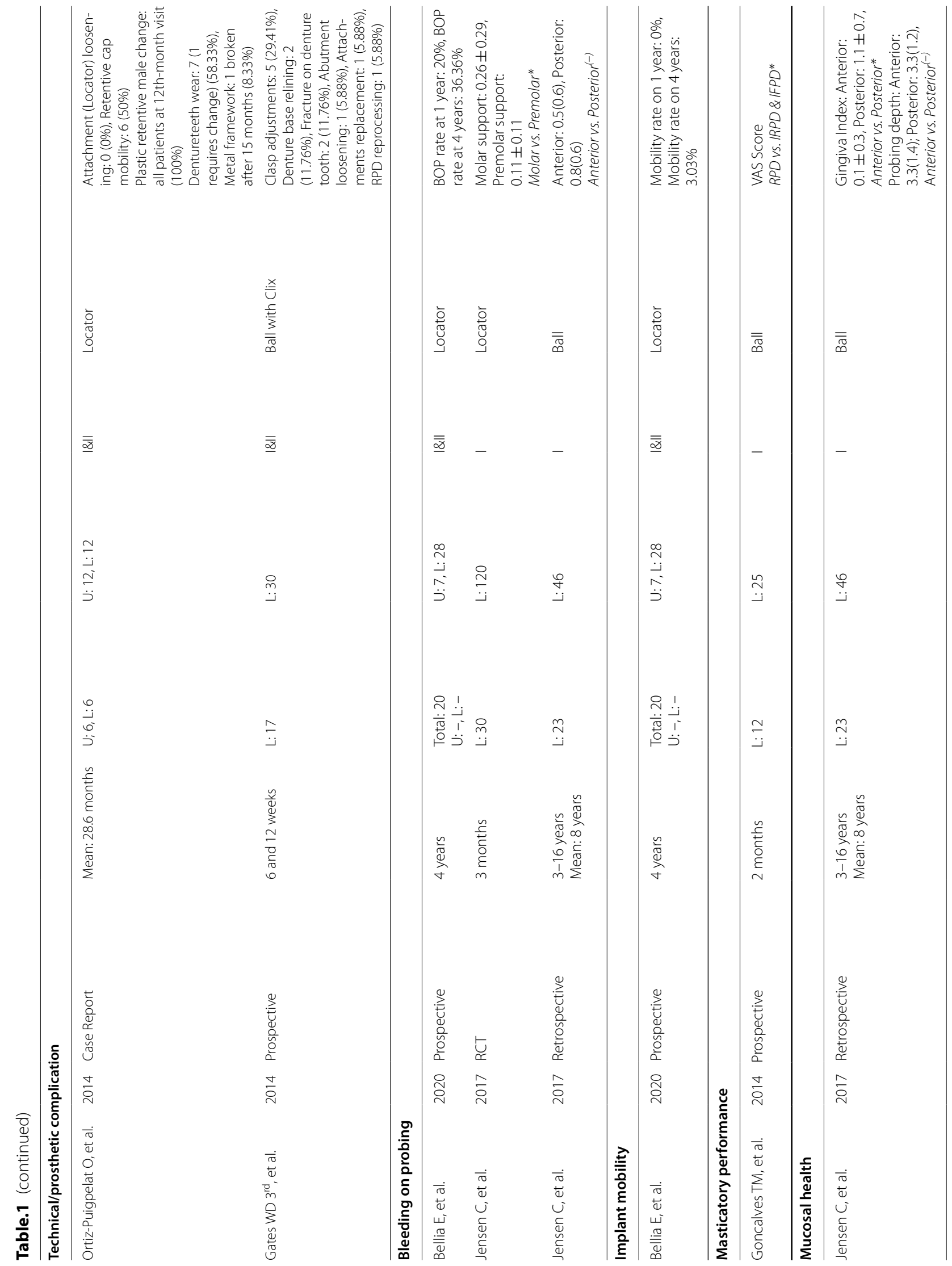




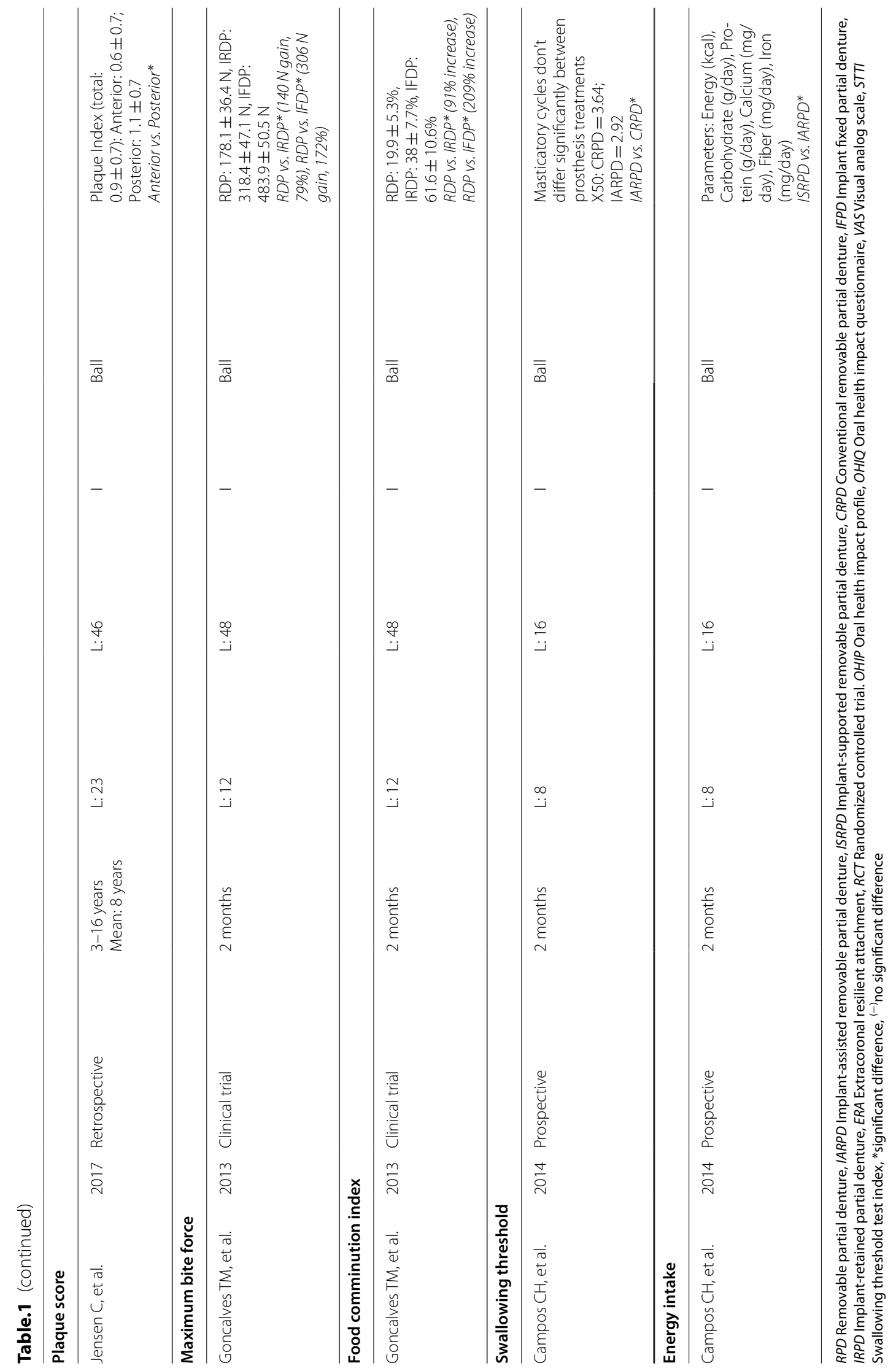


one RPD needed reprocessing and so on. [21]. Thus, the described technical/prosthetic complication data were very heterogeneous, since each study utilized different types of attachments. A more detailed description of studies reporting technical/prosthetic complications is provided in Table 1.

\section{Other clinical outcomes}

Other clinical outcomes, such as the occlusal contact area, occlusal force, bleeding on probing, implant mobility, masticatory performance, mucosal health, plaque score, maximum masticatory force, food comminution index, swallowing threshold, and energy intake were also reported. Jensen et al. reported significantly higher bleeding on probing rates with implants placed in the molar region compared to the premolar region [26]. Suzuki et al. [31] and Ohkubo et al. [16] found a significantly higher occlusal force and occlusal contact area with ISRPD/IARPD compared to CRPD. Goncalves et al. reported that IARPD exhibited a significantly higher masticatory performance, maximal masticatory force, and food comminution index than those for CRPD [22]. Jensen et al. reported significantly better gingival and plaque indices for more anteriorly placed implants compared to those placed posteriorly [28]. Campos et al. stated that IARPD yielded a significantly better result with respect to the swallowing threshold and energy intake assessment compared to CRPD [20].

\section{Discussion}

The terminology used by previous studies on ISRPD/ IARPD lacked uniformity, even though they described similar oral conditions and prosthetic designs. We opine that the terminology for implant-based RPDs should depend on the nature of the implants' function (support, retention, or bracing). Healing abutments only provide support in implant-supported dentures, without providing retention. On the other hand, attachments perform both functions. We considered the term "implant-assisted removable partial dentures" to be the most suitable, since our review focused on the differences between the attachment systems.

The treatment outcomes of IARPD can be compared with other treatment modalities, such as implantsupported fixed dental prostheses (ISFDP) and IOD. Pjetursson et al. reported that the implant survival rate of ISFDP was $95.6 \%(94.4-96.6 \%)$ at 5 years and $93.1 \%$ (90.5-95.0\%) at 10 years, although the success rate of prostheses without complications was only $66.4 \%$ over 5 years [34]. The implant survival rates of IOD were $73-100 \%$ in the maxilla [35], and $71-100 \%$ for the maxillary and mandibular arches [36]. Our review found that the implant survival rate of IARPD ranged between 91.6 and $100 \%$ for the maxillary and mandibular arches. A comparison revealed that the survival rate of IARPD was acceptable, when compared to that of IOD and ISFDP, although it was not proven statistically. The disparity in the study design and evaluation period did not permit the performance of a meta-analysis in this study.

A systematic review and meta-analysis conducted by Borges et al. revealed that the ISFDP and IOD differed only with respect to the oral health-related quality of life and satisfaction, although the ISFDP tended to show comparatively better results [37]. However, other indicators, such as implant survival rate, marginal bone loss, and periodontal diseases did not show that ISFDP was more efficient than IOD. This observation could be attributed to the greater stability of fixed prostheses, which was probably responsible for better patient satisfaction [9]. Nevertheless, ISFDP is not always the best treatment choice for all patients, especially considering economic factors, availability of inter-occlusal space, remaining bone volume, and maintenance of implants and prostheses. To overcome those circumstances, IARPD can be chosen. The results of this study indicated significantly greater patient satisfaction with IARPD compared to that with CRPD or before IARPD treatment, which confirms the findings of previous reviews $[10,11,38]$.

This distribution of the included studies in this review that analyzed the clinical outcomes based on the Kennedy classification was as follows: 14 studies included class I cases [16, 19, 20, 22-28, 30-33], 4 studies included class I and II cases [15, 18, 21, 29], and only one study focused on class II cases alone [17]. However, we could not compare the data of studies that incorporated a mix of class I and II cases, since the results did not clearly differentiate between the data for class I and class II in these studies. Two outcomes were compared (Figs. 2 and 3), which illustrate that the implant survival rate of IARPD for Kennedy's class I (91.6-100\%) was lower than that for Kennedy's class II (100\%). However, only a brief comparison was possible owing to the disparity in the sample size and study design between the two groups. The class II group also presented with a slightly lower level of mean bone loss $(0.3 \mathrm{~mm})$ than that in the class I group $(0.47-2.20 \mathrm{~mm})$. The nature of edentulism in Kennedy's class I, in which fewer remaining teeth are available to provide retention and support, may result in greater instability of the CRPD and the transmission of higher lateral stress to the implants. Moreover, Resnik stated that Kennedy's class I patients who have a higher risk of biological or prosthodontic complications need more implant support compared to most class II or III patients [39]. Biomechanically, the purpose of placing the implant with IARPD in Kennedy's class I and II conditions is to 
Implant Survival Rate (\%)

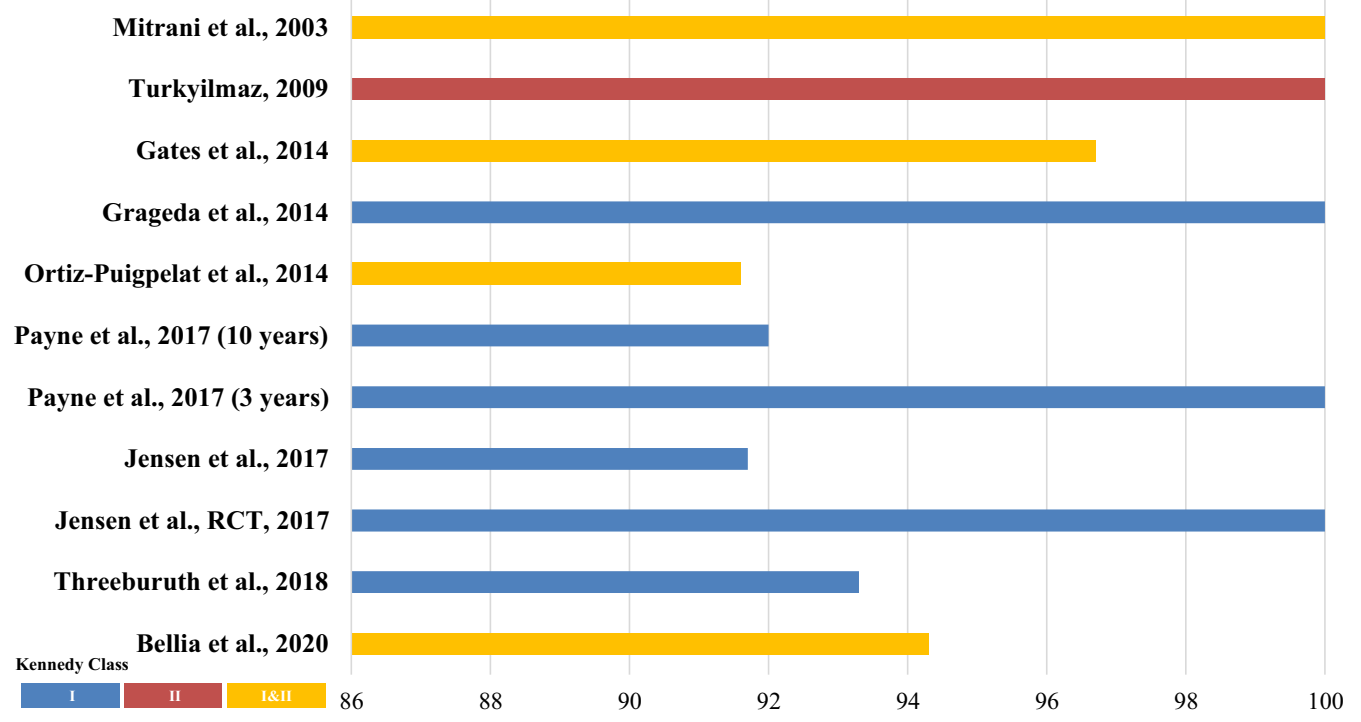

Fig. 2 Comparison of the implant survival rate in the included studies based on Kennedy's classification of the study samples [*Payne et al. reported the survival rates for 3 years and 10 years, separately [30]]

convert an unstable, distal extension edentulous area that lacks support to a class III configuration with greater support and retention, reduces the torque on the abutment tooth, and minimizes the need for clasps in the RPD design $[9,40]$.

The attachment system is among the factors that can influence the outcomes of IARPD, since different attachment systems possess different characteristics and mechanisms. Most of the included studies utilized stud-type attachments (18 of 19 studies, 94.7\%). The remaining study [16] used a rounded healing abutment as support. Based on the retentive mechanism, the attachment system was classified into the stud (O-ring, extra-coronal resilient attachment, ball, locator, and magnet), bar, and telescopic attachments. The stud attachment is further subclassified into the resilient and non-resilient types based on its function [12]. The ball attachment was found to be the most used attachment in the included studies, since this type of attachment is simple, cost effective, and less technique-sensitive [41].

Most clinical studies reported favorable results, despite various differences in the attachments. According to Aldhohrah et al., the implant survival rate was high both on immediate and delayed loading, irrespective of the attachment type [13]. Data on patient satisfaction outcomes from all included studies was significantly better with IARPD use, irrespective of the utilization of the stud or healing abutment in the attachment system. This result emphasizes the findings of Kim et al's systematic review, i.e., patient satisfaction might be independent of the attachment system used [42]. Previous reviews also showed that IARPD increased patient satisfaction despite various attachments $[10,11,38]$. Furthermore, Goto et al. investigated the effect of attachment installation conditions on the load transfer and denture movements of IOD for three types of attachments. The attachment system and its method of installation both affected the load distribution between the implants and mucosa [43].

Mitrani et al. compared the outcomes between different types of attachment (healing abutment and resilient attachment) in their retrospective study but found no significant difference between these groups after measurement on the mesial or distal side [15]. Chen et al. mentioned that the height of the implant abutment, which differs for each attachment type, influences early bone loss around the implants [44]. Each of the three studies $[21,28,29]$ that evaluated the technical or prosthetic complication-related outcomes used different type of attachments, and the reported complications were too diverse and inconclusive. The most recent systematic review and meta-analysis on attachment systems in IOD were also unable to arrive at a definite conclusion due to the heterogeneity of the reported outcomes. The attachment system used in a prosthesis may influence prosthetic maintenance and complications [42]. Therefore, further investigations on this aspect are recommended. 


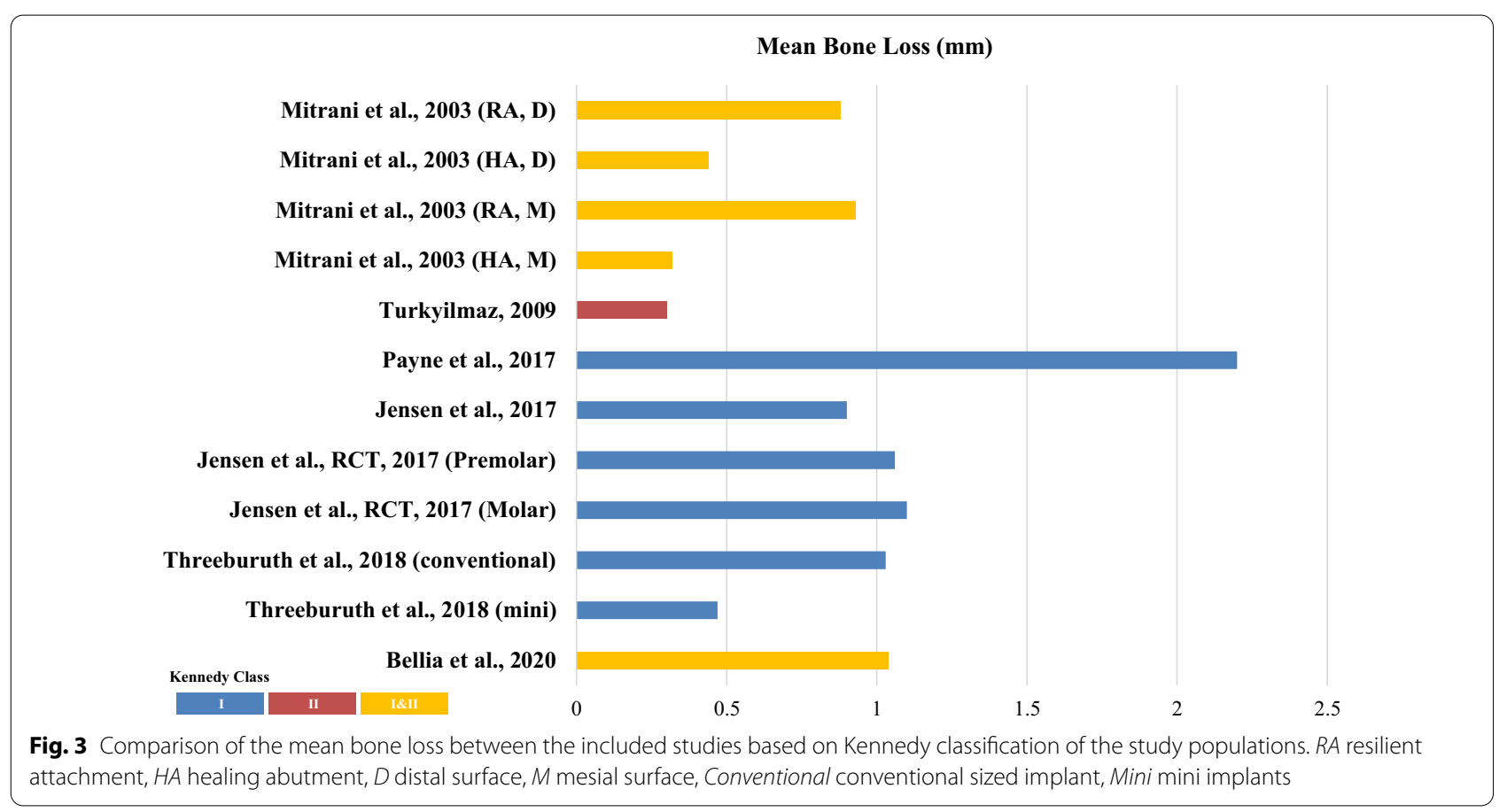

\section{Study limitations}

Although the overall results of the included studies showed favorable clinical outcomes, we could not analyze them statistically owing to the high degree of heterogeneity in the design, method, number of patients, and evaluation period. Regarding the evaluation period, Pandolfi et al. mentioned that peri-implant complications tend to occur after 5 years post-loading condition [45]. However, among 19 included studies, there was a follow-up for more than 5 years in only 2 studies $[28,30]$. For evidencebased treatment using IARPD, further reports with longterm follow-up should be accumulated.

Ideally, case reports should not be involved in a systematic review. However, there is a lack of published studies that fit the purpose of this study. Moreover, the reported clinical outcomes are also diverse. Goodacre et al. (2003) had comprehensively reviewed complications of implantsupported prostheses and included case reports [46]. Considering the lack of evidence regarding treatment outcomes based on the attachment system and Kennedy classification in distal extension IARPD, we conducted a comprehensive review and included those three case reports in our study.

Unfortunately, we could not perform a meta-analysis due to the lack of studies with a high quality of evidence. Among the 19 included studies, there were only 3 RCTs as the highest evidence level. Those studies also have various properties in terms of patient numbers, only evaluated in a short period (3, 6, 12 months) which is insufficient for outcomes. Moreover, there is a probability of patient allocation bias in clinical studies. At this point, a more homogenous or standardized protocol for further high-quality RCTs is recommended to facilitate the comparison of the clinical outcomes based on the different kinds of attachments. In addition to attachment systems, other variables, such as implant size (mini, conventional), and implant position which also contribute to IARPD success rate should be investigated further.

\section{Conclusion}

Within the limitations encountered in this literature review, it can be concluded that IARPD is among the viable prosthodontic treatment options for distal extension edentulous areas, which can yield favorable clinical outcomes. Although slight differences were observed between the implant survival rate and mean bone loss in Kennedy's class I and II, the comparison was not balanced owing to the variations in the study design, number of implants evaluated, and sample size. The stud attachment, especially the ball type, was used most commonly in IARPD treatment, since it is considered to be a simple, economical option, with favorable biological treatment outcomes. The use of different attachment systems overall did not significantly influence implant survival rate and patient satisfaction; however, this aspect and other clinical outcomes should be evaluated statistically, which necessitates the performance of more highquality studies. 


\section{Abbreviations}

RPD: Removable partial denture; IARPD: Implant-assisted removable partial denture; ISRPD: Implant-supported removable partial denture; CRPD: Conventional removable partial denture; ISFPD: Implant-supported fixed partial denture; IRPD: Implant-retained partial denture; ERA: Extracoronal resilient attachment; RCT: Randomized controlled trial.

\section{Acknowledgements}

Not applicable.

\section{Authors' contributions}

AYPW and TG are responsible for the study conception and design, acquisition of data, analysis, and interpretation of data, drafting of the manuscript, and critical revision. $\mathrm{Yl}, \mathrm{YI}$ and $\mathrm{MW}$ are for the critical revision. $\mathrm{Tl}$ is responsible for the study conception and design and critical revision. All authors read and approved the final manuscript.

\section{Funding}

Not applicable.

\section{Availability of data and materials}

The data sets generated and analysed during current study are available from the corresponding author upon reasonable request.

\section{Declarations}

\section{Ethics approval and consent to participate}

Not applicable.

\section{Consent for publication}

Not applicable.

\section{Competing Interests}

All authors declare that they have no competing interests.

Received: 28 April 2021 Accepted: 7 November 2021

Published online: 13 November 2021

\section{References}

1. Feine JS, Carlsson GF, Awad MA, Chehade A, Duncan WJ, Gizani S, et al. The McGill consensus statement on overdentures. Chicago: The Gerodontology Association; 2002.

2. Chikunov I, Doan P, Vahidi F. Implant-retained partial overdenture with resilient attachments. J Prosthodont. 2008:17(2):141-8.

3. Schwendicke J, Nitschke I, Stark H, Micheelis W, Jordan RA. Epidemiological trends, predictive factors, and projection of toot loss in Germany 1997-2030: part II. Edentulism in seniors Clin Oral Invest 2020;24:3997-4003.

4. Anon. Oral Health Surveillance report. Centers of Disease Control and Prevention. 2019. https://www.cdc.gov/oralhealth/publications/OHSR2019-summary.html. Accessed 26 Nov 2020

5. Curtis D, Curtis T, Wagnild G, Finzen F. Incidence of various classes of removable partial dentures. J Prosthet Dent. 1992;67:664-7.

6. Bilhan $\mathrm{H}$. Complication rates and patient satisfaction with removable dentures. J Adv Prosthodont. 2012:4:109-15.

7. Mijiritsky E. Implants in conjunction with removable partial dentures: a literature review. Implant Dent. 2007;16:146-54.

8. Carr A, Brown D. McCracken's removable partial prosthodontics. 12th ed. USA: Elsevier; 2011

9. Phoenix R, Cagna DR, DeFreest C. Stewart's clinical removable partial prosthodontics. 4th ed. India: Quintessence Books; 2014.

10. De Freitas RFCP, Dias KD, Carreiro ADFP, Barbosa GAS, Ferreira MAF Mandibular implant-supported removable partial denture with distal extension: a systematic review. J Oral Rehabil. 2012;39(10):791-8.

11. Park J, Lee J, Shin S, Kim H. Effect of conversion to implant-assisted removable partial denture in patients with mandibular Kennedy classification I: a systematic review and meta-analysis. Clin Oral Impl Res. 2020:31:360-73.
12. Ahmed Y. Attachments used with implant supported over denture. Adv Dent Oral Health. 2016;1(2):45-8.

13. Aldhohrah T, Mashrah MA, Wang Y. Effect of 2 implant-implant mandibular overdenture with different attachments and loading protocols on peri-implant health and prosthetic complications: a systematic review and network meta-analysis. J Prosthet Dent. 2021. https://doi.org/10. 1016/j.prosdent.2020.12.016.

14. Moher D, Liberati A, Tetzlaff J, Altman DG, PRISMA Group. Preferred reporting items for systematic reviews and meta-analyses: the PRISMA statement. Ann Intern Med. 2009;151:264-9.

15. Mitrani R, Brudvik J, Philips K. Posterior implants for distal extension removable prostheses: a retrospective study. Int J Periodontics Restorative Dent. 2003;23(4):353-9.

16. Ohkubo C, Kobayashi M, Suzuki Y, Hosoi T. Effect of implant support on distal-extension removable partial dentures: in vivo assessment. Int J Oral Maxillofac Implants. 2008;23:1095-101.

17. Turkyilmaz I. Use of dental implants to support and increase retention of a removable partial denture: a case report. JCDA. 2009;75(9):655-8.

18. Bellia E, Audenino G, Ceruti P, Bassi F. Clinical assessment of short implants retaining removable partial dentures. Int J Oral Maxillofac Implants. 2020;35(1):207-13.

19. Campos CH, Goncalves TMSV, Garcia RCMR. Implant-supported removable partial denture improves the quality of life of patients with extreme tooth loss. Braz Dent J. 2015;26(5):463-7.

20. Campos C, Goncalves T, Matheus R, Garcia R. Implant retainers for freeend removable partial dentures affect mastication and nutrient intake. Clin Oral Impl Res. 2014:25:957-61.

21. Gates WD, Cooper LF, Sanders AE, Reside GJ, De Kok IJD. The effect of implant-supported removable partial dentures on oral health quality of life. Clin Oral Impl Res. 2014;25:207-13.

22. Goncalves T, Campos C, Garcia C. Mastication and jaw motion of partially edentulous patients are affected by different implant-based prostheses. J Oral Rehabil. 2014:41:507-14.

23. Goncalves TMSV, Campos CH, Garcia RCMR. Implant retention and support for distal extension partial removable dental prostheses: satisfaction outcomes. J Prosthet Dent. 2014;112:334-9.

24. Goncalves TMSV, Campos CH, Moraes M, Rodrigues Garcia RCM. Mastication improvement after partial implant-supported prosthesis use. J Dent Res. 2013;92(Suppl 12):189S-S194.

25. Grageda E, Rieck B. An implant-assisted removable partial denture: a 36-month follow-up case report. Dent Today. 2014;17:58.

26. Jensen C, Speksnijder CM, Raghoebar GM, Kerdijk W, Meijer HJA, Cune MS. Implant-supported mandibular removable partial dentures: Functional, clinical, and radiographical parameters in relation to implant position. Clin Implant Dent Relat Res. 2017;19:432-9.

27. Jensen C, Meijer HJA, Raghoebar GM, Kerdijk W, Cune MS. Implant-Supported Mandibular Removable partial dentures; patient-based outcome measures in relation to implant position. J Prosthodont Res. 2016. https:// doi.org/10.1016/j.jdent.2016.10.008.

28. Jensen C, Meijer HJA, Raghoebar GM, Kerdijk W, Cune MS. Implantsupported removable partial dentures in the mandible: a 3-16 years retrospective study. J Prosthodont Res. 2016. https://doi.org/10.1016/j. jpor.2016.07.002.

29. Ortiz-Puigpelat O, Gargallo-Albiol J, Hernandez-Alfaro F, Cabratosa-Termas J. Short-term retrospective case series of implant-assisted removable partial dentures with locator abutments. Int J Periodontics Restorative Dent. 2014;34(6):121-8

30. Payne AG, Tawse-Smith A, Wismeijer D, De Silva RK, Ma S. Multicentre prospective evaluation of implant-assisted mandibular removable partial dentures: surgical and prosthodontic outcomes. Clin Oral Impl Res. 2017;28:115-25.

31. Suzuki Y, Kno K, Shimpo H, Sato Y, Ohkubo C. Clinical evaluation of implant-supported removable partial dentures with a stress-breaking attachment. Implant Dent. 2017;26(4):516-23.

32. Threeburuth W, Aunmeungtong W, Khongkhunthian P. Comparison of immediate-load mini dental implants and conventional-size dental implants to retain mandibular Kennedy class I removable partial dentures: a randomized clinical trial. Clin Implant Dent Relat Res. 2018;20:785-92. 
33. Wismeijer D, Tawse-Smith A, Payne AGT. Multicentre prospective evaluation of implant-assisted mandibular bilateral distal extension removable partial dentures: Patient satisfaction. Clin Oral Impl Res. 2011;00:1-8.

34. Pjetturson BE, Thoma D, Jung R, Zwahlen M, Zembic A. A systematic review of survival and complication rates of implant-supported fixed dental prostheses (FDPs) after mean observation period of at least 5 years. Clin Oral Impl Res. 2012. https://doi.org/10.1111/j.1600-0501.2012. 02546.x.

35. Sadowsky SJ. Treatment consideration for maxillary implant overdentures: a systematic review. J Prosthet Dent. 2007;97:340-8.

36. Andreiotelli M, Att W, Strub JR. Prosthodontic complications with implant overdentures: a systematic review. Int J Prosthodont. 2010;23:195-203.

37. Borges GA, Barbin T, Dini C, Maia LC, Magno BM, Barao VAR, et al. Patient-reported outcome measures and clinical assessment of implantsupported overdentures and fixed prostheses in mandibular edentulous patients: a systematic review and meta-analysis. J Prosthet Dent. 2020. https://doi.org/10.1016/j.prosdent.2020.11.005.

38. Zancope K, Abrao G, Karam F, Neves F. Placement of a distal implant to convert a mandibular reovable Kennedy Class I to an implant-supported partial removable Class III dental prosthesis: a systematic review. J Prosthet Dent. 2015;113(6):528-33.

39. Resnik RR. Misch's contemporary implant dentistry. 4th ed. Canada: Elsevier; 2020

40. Grossmann Y, Nissan J, Levin L. Clinical effectiveness of implantsupported removable partial dentures-a review of the literature and retrospective case evaluation. J Oral Maxiloffac Surg. 2009;67:1941-6.
41. Krennmair G, Weinlander M, Krainhofner M, Piehslinger E. Implantsupported mandibular overdentures retained with ball or telescopic crown attachments: a 3-year prospective study. Int J Prosthodont. 2006;19(2):164-70.

42. Kim H, Lee J, Shin S, Bryan S. Attachment systems for mandibular implant overdentures: a systematic review. J Adv Prosthodont. 2012;4(4):197-203.

43. Goto T, Nagao K, Ishida Y, Tomotake Y, Ichikawa T. Influence of matrix attachment installation load on movement and resultant forces in implant overdentures. J Prosthodont. 2015;24:153-6.

44. Chen Z, Lin C, Li J, Wang H, Yu H. Influence of abutment height on periimplant marginal bone loss: a systematic review and meta-analysis. J Prosthet Dent. 2019;122:14-21.

45. Pandolfi A, Rinaldo F, Pasqualotto D, Sorrentino F, Torre GL, Guerra F. A Retrospective cohort study on peri-implant complications in implants up to 10 years of functional loading in periodontally compromised patients. J Periodontol. 2020;91:995-1002.

46. Goodacre CJ, Bernal G, Rungcharassaeng K, Kan JYK. Clinical complications with implants and implant prostheses. J Prosthet Dent 2003;90:121-32.

\section{Publisher's Note}

Springer Nature remains neutral with regard to jurisdictional claims in published maps and institutional affiliations.

\section{Submit your manuscript to a SpringerOpen ${ }^{\circ}$ journal and benefit from:}

- Convenient online submission

- Rigorous peer review

- Open access: articles freely available online

- High visibility within the field

- Retaining the copyright to your article

Submit your next manuscript at $\boldsymbol{\nabla}$ springeropen.com 